\title{
Avaliação Microestrutural e Propriedades Mecânicas de um Aço TRIP Soldado a Ponto por Fricção e Mistura Mecânica
}

\author{
(Microstructural Evaluation and Mechanical Properties of a Friction Stir Spot Welded TRIP 800 Steel)
}

\begin{abstract}
Cíntia Cristiane Petry Mazzaferro ${ }^{1,2}$, Fabiano Dornelles Ramos ${ }^{1,2}$, José Antônio Esmerio Mazzaferro ${ }^{3}$, Tonilson de Souza Rosendo ${ }^{1,2}$, Marco Antônio Durlo Tier ${ }^{1,4}$, Antônio Mônaco da Silva ${ }^{5}$, Jorge Fernandez dos Santos ${ }^{1}$, Afonso Reguly ${ }^{2}$

${ }^{I}$ GKSS Research Centre, Institute of Materials Research, Materials Mechanics, Solid State Joining Processes, Geesthacht, Alemanha

${ }^{2}$ Universidade Federal do Rio Grande do Sul-UFRGS, DEMET, PPGEM, Porto Alegre, Brasil

${ }^{3}$ Universidade Federal do Rio Grande do Sul-UFRGS, DEMEC, PROMEC, Porto Alegre, Brasil

${ }^{4}$ Universidade Regional Integrada do Alto Uruguai e das Missões-URI, DECC, EIM, Santo Ângelo, Brasil

${ }^{5}$ anteriormente no GKSS Research Centre, Institute of Materials Research, Materials Mechanics, Solid State Joining Processes, atualmente no Centro de Investigación en Tecnologías de Unión - LORTEK, Ordizia, Espanha

cmazzaferro@demet.ufrgs.br
\end{abstract}

\section{Resumo}

Neste estudo, um aço TRIP 800 foi soldado através do processo SPFMM usando três diferentes velocidades de rotação da ferramenta, 1600, 2000 e 2400 rpm, e mantendo-se o tempo de mistura fixo em 2s. Foram mostradas as microestruturas resultantes em cada zona formada, assim como a variação de dureza através delas. Maiores valores de dureza foram obtidos na menor velocidade de rotação, 1600 rpm, onde o aporte de energia teoricamente foi menor e, portanto, a taxa de resfriamento foi maior. Porém, nesta velocidade de rotação também houve formação de ferrita alotriomorfa na microestrutura. Nos ensaios de cisalhamento, amostras soldadas nas velocidades de 1600 rpm e 2400 rpm não atingiram o valor de força mínimo recomendado pela norma AWS D8.1M, o que foi atribuído ao menor comprimento da linha de ligação e à menor distância entre a parede do furo deixado pelo pino e a linha de zinco formada na zona de mistura, uma vez que durante a fratura a trinca propaga através desta linha e a microestrutura só possui algum papel no processo de fratura quando ela não existe mais.

Palavras-chave: microestrutura; propriedades mecânicas; soldagem a ponto por fricção e mistura mecânica; aço TRIP.

\begin{abstract}
A TRIP 800 steel was friction stir spot welded using three different tool rotational speeds, 1600, 2000 and 2400 rpm, and the dwell time was kept constant in $2 s$. The resultant microstructures formed in each weld zone were analyzed, as well as their hardness. Higher hardness values were observed for the lowest rotational speed, 1600 rpm, where the heat input in theory was lower and, therefore, the cooling rate was faster. However, for this rotational speed allotriomorphic ferrite was also observed in the stir zone. In the lap-shear tests, samples welded at $1600 \mathrm{rpm}$ and $2400 \mathrm{rpm}$ did not reach the minimum value recommended by the AWS D8.1M standard, which was attributed to the lower bonding ligament length and also lower distance between the keyhole left by the pin and the end of the zinc line, which is formed in the stir zone. The fracture of the samples occurred along this line. As a result the influence of the microstructure on the failure process could only be inferred when the zinc line disappeared.
\end{abstract}

Key-words: microstructure; mechanical properties; friction stir spot welding; TRIP steel.

\section{Introdução}

Os aços avançados de alta resistência foram desenvolvidos para uso na indústria automobilística visando redução de peso e economia de combustível, sem comprometer a segurança dos passageiros. Estes aços apresentam resistências superiores a 500 MPa com boa ductilidade, e incluem aços como os dual-phase (DP), complex-phase (CP), martensíticos (M ou MART) e os

(Recebido em 7/12/2008; Texto Final em 09/11/2009). que apresentam o efeito de plasticidade induzida por deformação (TRIP). A utilização de aços avançados de alta resistência em alguns veículos (através da substituição não só de material, mas de projeto de componentes) pode gerar reduções de peso em pelo menos $20 \%$ [1,2], com melhor desempenho em testes de colisão e sem aumentos de custos.

Os aços TRIP têm despertado grande interesse na indústria automotiva pelas suas características de alta resistência, boa conformabilidade e alta capacidade de absorção de energia. Eles possuem microestrutura composta por ferrita, bainita e austenita retida. Esta microestrutura é obtida através de adição de elementos de liga como manganês e silício e de tratamento 
térmico realizado em duas etapas: recozimento intercrítico seguido de tratamento isotérmico no campo bainítico [3-6]. Durante o tratamento isotérmico ocorre transformação parcial da austenita em bainita, enriquecendo a austenita remanescente em carbono, o que a estabiliza contra a transformação martensítica durante posterior resfriamento. Porém, quando o aço TRIP é deformado plasticamente ocorre transformação gradual da austenita em martensita, evitando a estricção e resultando em maior alongamento uniforme $[7,8]$.

No entanto, devido à adição de elementos de liga, o carbono equivalente de aços TRIP pode ser elevado, e conseqüentemente a sua soldagem pode ser problemática. Os processos de soldagem mais empregados na indústria automobilística são soldagem a ponto por resistência elétrica e soldagem a laser. Devido às altas temperaturas atingidas durante o processo, com fusão do material, as taxas de resfriamento associadas a estes processos são elevadas, o que leva à formação durante o resfriamento de estruturas martensíticas, duras e frágeis. Quando o componente soldado é posteriormente submetido a cargas, podem ocorrer fraturas frágeis interfaciais. Para contornar este problema, existem alguns estudos especificamente sobre aços TRIP sugerindo algumas adequações ao processo, tais como emprego de revenimento durante $[9,10]$ ou pós-solda [11]. Outro fator que pode dificultar a soldagem de aços TRIP - e também de outros aços - é a presença de zinco na superfície da chapa, empregado para conferir resistência à corrosão ao material [12,13]. Em soldas a ponto por resistência elétrica, a vida útil dos eletrodos é diminuída devido às reações químicas que ocorrem entre o zinco e o cobre do eletrodo, além da utilização de forças maiores para soldagem. O zinco possui baixo ponto de fusão (cerca de $420^{\circ} \mathrm{C}$ ) e sua temperatura de vaporização é de aproximadamente $907^{\circ} \mathrm{C}$. Estas temperaturas são facilmente atingidas na soldagem de aços, e se vapores de zinco ficarem aprisionados no metal de solda fundido, resultará em porosidades. Para que isto seja evitado em soldas a laser é procedimento comum deixar um pequeno espaço entre as chapas a serem soldadas [10,14].

Sendo assim, a utilização de soldagem a ponto por fricção e mistura mecânica (SPFMM) como processo de união tornase atrativa, pois as temperaturas atingidas durante o processo não excedem a temperatura de fusão do material. Na SPFMM, o calor gerado por uma ferramenta em rotação aquece os materiais, plastificando-os e misturando-os mecanicamente. O processo consiste em três etapas, como mostrado na Figura 1 [15]. Na primeira etapa, a ferramenta em rotação, composta por pino e ombro, é forçada contra as chapas a serem soldadas sob uma determinada pressão. Esta pressão cria calor de fricção entre as chapas e o pino, plastificando o material das chapas. O pino penetra totalmente nas chapas, até que o ombro toca a superfície da chapa superior. Quando o pino atinge uma profundidade préestabelecida nas chapas, a ferramenta é mantida em rotação durante um determinado período de tempo, plastificando e misturando mecanicamente os materiais das chapas, etapa 2; e finalmente na última etapa a ferramenta é retirada. Ao término do processo, remanesce na junta um "furo" com o perfil do pino.

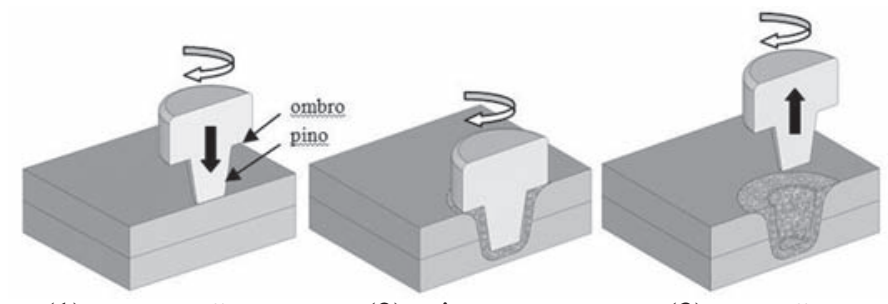

(1) penetração

(2) mistura

(3) retração

Figura 1. Etapas do processo de SPFMM. Adaptado de Mazzaferro et al. [15].

Ao processo de SPFMM estão associadas não apenas vantagens técnicas, mas também econômicas: a substituição da soldagem a ponto por resistência pela Mazda gerou uma economia de energia de $99 \%$ em ligas de alumínio e de $80 \%$ em ligas de aço e redução de investimentos em equipamentos em $40 \%$ para ambos os materiais [16]. Já existem alguns estudos sobre o emprego de SPFMM em aços avançados de alta resistência [17-20], porém eles não incluem aços TRIP.

Sendo assim, este trabalho teve por objetivo verificar a possibilidade de obtenção de juntas de um aço TRIP 800 através de SPFMM, e correlacionar as microestruturas com propriedades mecânicas resultantes. Além da caracterização microestrutural, ensaios de microdureza e de cisalhamento foram efetuados para entender o comportamento mecânico das juntas.

\section{Materiais e Métodos}

Neste estudo foi utilizado um aço TRIP 800 laminado a frio, com espessura de 1,0 mm e revestimento de zinco de 7,5 $\mu \mathrm{m}$ em ambos os lados. Sua composição química é mostrada na Tabela 1.

Tabela 1. Composição química do aço TRIP empregado (\% em massa).

\begin{tabular}{cccccccc}
\hline $\mathrm{C}$ & $\mathrm{Mn}$ & $\mathrm{Si}$ & $\mathrm{Al}$ & $\mathrm{P}$ & $\mathrm{S}$ & $\mathrm{N}$ & $\mathrm{Ti}$ \\
\hline 0,19 & 1,64 & 1,65 & 0,039 & 0,016 & 0,0009 & 0,0023 & 0,0097 \\
\hline
\end{tabular}

As juntas foram produzidas em um pórtico especialmente projetado para executar soldas por fricção e mistura mecânica. Foi usado controle de deslocamento, ou seja, a profundidade de penetração da ferramenta era pré-estabelecida em 1,6 mm, para então ser aplicado o tempo de mistura. A ferramenta produzida em nitreto cúbico de boro policristalino (PCBN) possuía um diâmetro cônico de $15 \mathrm{~mm}$, assim como um pino cônico com diâmetro menor de $5 \mathrm{~mm}$ e comprimento de 1,5 mm. O pino possuía seis facetas igualmente espaçadas. As velocidades de soldagem empregadas foram 1600, 2000 e $2400 \mathrm{rpm}$, e o tempo de mistura utilizado foi de $2 \mathrm{~s}$.

A caracterização microestrutural das amostras foi realizada em microscópio ótico (MO) e em microscópio eletrônico de varredura (MEV), em amostras preparadas seguindo o procedimento metalogáfico desenvolvido por Mazzaferro [21] e, após, atacadas com Nital 1\% ou com LePera. O uso deste último reagente facilitou a identificação de algumas fases, pois após este 
ataque a ferrita tem cor azul, a bainita cor marrom e a austenita e a martensita, cor branca [22]. Porém, o LePera foi sempre usado em amostras analisadas no MO, preferindo-se utilizar ataque com Nital nas amostras analisadas no MEV porque o contraste entre as fases era melhor. Outro fato que facilitou a identificação das diferentes fases formadas no processo, além de suas cores após ataque com LePera, foi suas localizações e morfologias: por exemplo, a ferrita alotriomorfa e a ferrita de Widmanstätten possuem cor azulada após o uso de LePera, porém enquanto que a ferrita alotriomorfa nucleia diretamente a partir dos contornos de grãos austeníticos e possui forma lenticular ou equiaxial, a ferrita de Widmanstätten, por sua vez, nucleia geralmente a partir da ferrita alotriomorfa e possui a forma de placas [23].

A variação de dureza através das juntas soldadas foi determinada através de ensaios de microdureza Vickers, que foram realizados nas seções transversais das amostras aplicando uma carga de $0,5 \mathrm{~kg}$ durante $10 \mathrm{~s}$. A distância entre identações foi de $0,25 \mathrm{~mm}$.

A resistência das juntas foi avaliada através de ensaios de cisalhamento; como não existe norma específica para processos de SPFMM, foi utilizada a norma para soldas a ponto por resistência elétrica ISO 14273 [24]. Foram ensaiados três corpos de prova para cada condição estudada. As superfícies de fratura foram analisadas no MEV e, após, estas amostras cisalhadas foram cortadas na direção do carregamento aplicado; suas superfícies foram preparadas metalograficamente e elas foram analisadas no MO, usando LePera como ataque químico, e no $\mathrm{MEV}$, usando ataque com Nital $1 \%$.

\section{Resultados e Discussão}

A microestrutura do metal base (MB) é mostrada na Figura 2. O aço TRIP possuía microestrutura de bainita e austenita retida em matriz ferrítica.

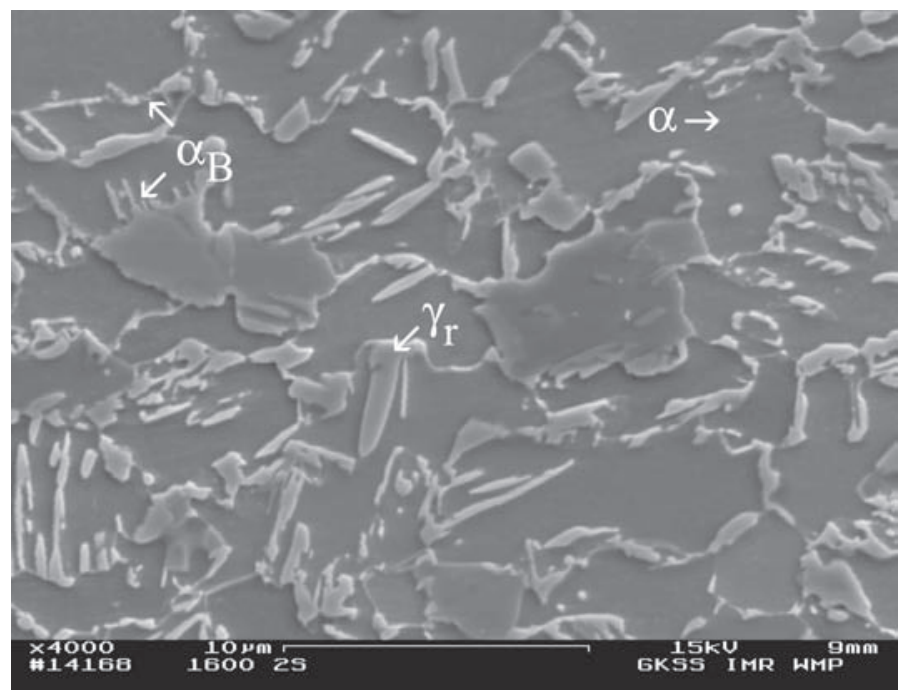

Figura 2. Microestrutura do metal base: ferrita $\alpha$, bainita $\alpha_{B}$ e austenita retida $\gamma_{r}$. MEV, ataque com Nital $1 \%$.

Devido aos ciclos térmicos e deformações impostas pelo processo, três diferentes zonas foram formadas nas juntas, como pode ser observado na Figura 3(a): a zona de mistura (ZM), onde a temperatura atingida e a deformação plástica causaram transformações microestruturais e mistura dos materiais das chapas superior e inferior; a zona termomecanicamente afetada (ZTMA), caracterizada por alto pico de temperatura atingido durante o processo juntamente com deformação plástica (mas esta menor do que na ZM), e onde pode ocorrer recristalização; e a zona termicamente afetada (ZTA), que não sofre nenhuma deformação plástica. A ZTA é dividida em suas subzonas, ZTA1 e ZTA2, onde ocorrem diferentes mudanças microestruturais devido ao gradiente térmico.

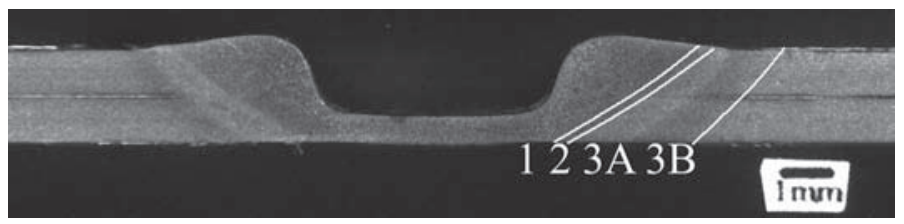

Figura 3. Macroestrutura mostrando localização das diferentes zonas fornadas após processamento: 1=zona de mistura (ZM), 2=zona termomecanicamente afetada (ZTMA); 3A, 3B= zona termicamente afetada (ZTA). Condição 1600 rpm - 2s. Ataque Nital $1 \%$.

Devido ao fluxo de material, o zinco presente no revestimento das chapas foi incorporado à $\mathrm{ZM}$, remanescendo uma linha de zinco após a soldagem; um exemplo pode ser visto na Figura 4. A geometria desta linha de zinco, o seu comprimento e a distância entre o seu final e a parede do furo deixado pelo pino, mostraram-se dependentes da velocidade de rotação usada.

Alguns autores [25, 26] citam a que resistência de juntas de aços DP produzidas através do processo SPFMM está relacionada ao comprimento da linha de ligação (CLL), ou seja, ao comprimento da região na qual não existe interface entre as chapas superior e inferior, porém naqueles trabalhos os aços utilizados não apresentavam revestimentos. Sendo assim, neste estudo foram medidos nas amostras usadas para análise metalográfica não apenas o comprimento da linha de ligação, mas também a distância entre a parede do furo deixado pelo pino e o final da linha de zinco (DPZ). Os resultados encontram-se na Tabela 2, onde se observa que os maiores valores de CLL e DPZ foram obtidos quando foi utilizada a velocidade de rotação de $2000 \mathrm{rpm}$.

Tabela 2. Comprimento da linha de ligação (CLL) e distância entre a parede do furo deixado pelo pino e o final da linha de zinco (DPZ) para cada velocidade de rotação*.

\begin{tabular}{cccc}
\hline $\begin{array}{c}\text { Velocidade de } \\
\text { rotação, rpm }\end{array}$ & 1600 & 2000 & 2400 \\
\hline CLL, $\mu \mathrm{m}$ & 489,6 & 747,3 & 649,1 \\
DPZ, $\mu \mathrm{m}$ & 249,0 & 382,1 & 269,1 \\
\hline
\end{tabular}

*média de três medições em cada lado da junta, em amostras sem ataque. 


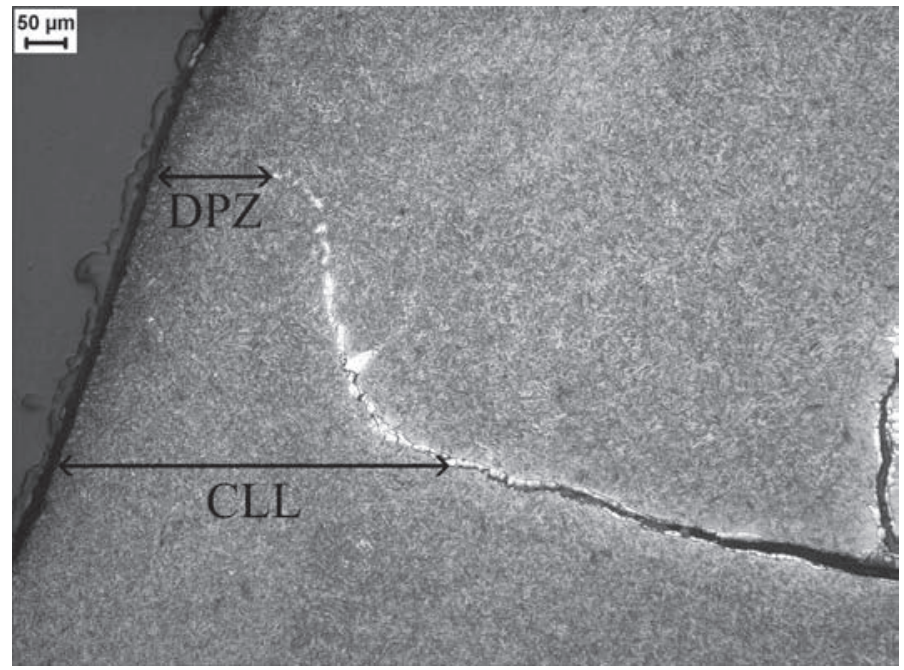

Figura 4. Aspecto da linha de zinco remanescente na ZM após o processo, mostrando a distância entre o seu final e a parede do furo deixado pelo pino (DPZ), assim como o comprimento da linha de ligação (CLL). Velocidade de rotação 2400 rpm. MO, ataque com LePera.

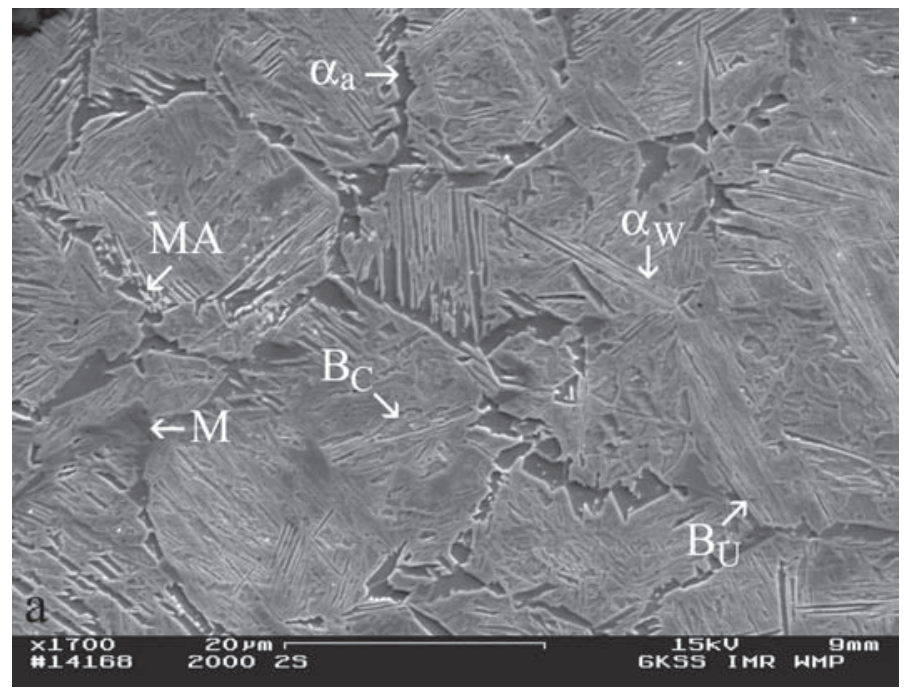

As microestruturas resultantes na ZM são mostradas na Figura 5; elas são compostas por uma mistura de ferrita alotriomorfa $\left(\alpha_{\mathrm{a}}\right)$, ferrita de Widmanstätten $\left(\alpha_{\mathrm{w}}\right)$, bainita $(\mathrm{B})$, bainita coalescida $\left(\mathrm{B}_{\mathrm{C}}\right)$, martensita $(\mathrm{M})$ e constituinte martensitaaustenita (MA). As quantidades de ferrita alotriomorfa, ferrita de Widmanstätten, martensita e martensita-austenita diminuíram da região mais próxima ao furo deixado pelo pino em direção ao meio da ZM, enquanto que as que as de bainita aumentaram.

Em todas as velocidades de rotação de ferramenta utilizadas houve a formação de ferrita alotriomorfa, principalmente em regiões adjacentes ao furo deixado pelo pino, e sua quantidade aumentou com o aumento da velocidade de rotação. Isto pode ser atribuído ao fato de que na SPFMM, um aumento da velocidade de rotação teoricamente levou a um maior aporte de energia e, conseqüentemente, menor taxa de resfriamento. Além disso, devido à maior temperatura atingida em maiores velocidades de rotação, a resistência do material é menor nestas condições, ou seja, ele se deforma mais facilmente. Estudos mostraram que o aumento da deformação leva a um aumento na quantidade de ferrita alotriomorfa, seja devido ao aumento na quantidade de fronteiras de grão de austenita, que são os lugares preferenciais para a nucleação da ferrita [2729], e/ou redução no super-resfriamento necessário para a sua nucleação [30, 31].

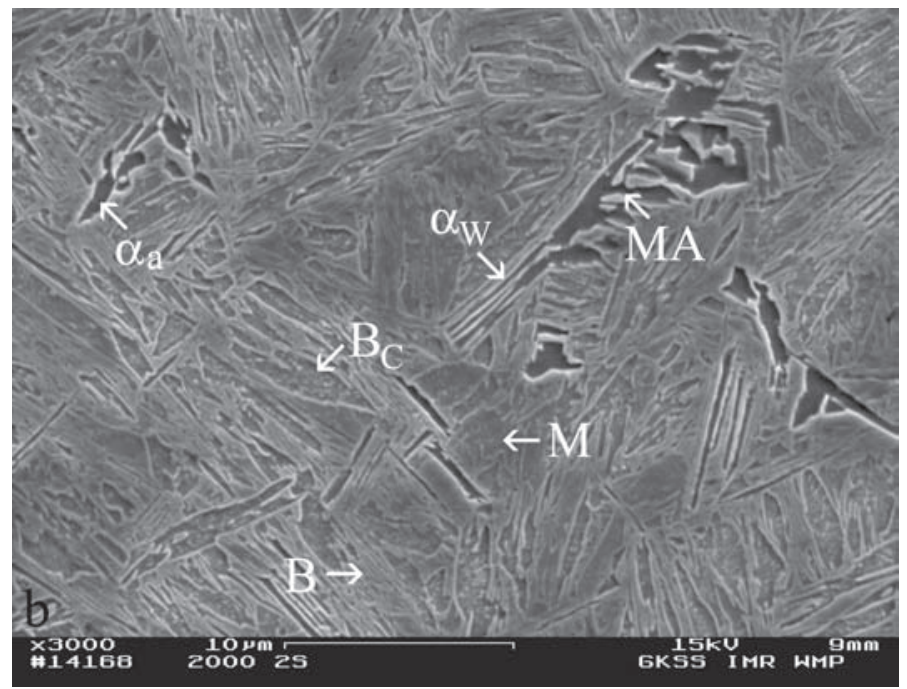

Figura 5. Microestruturas na ZM: (a) região adjacente ao furo deixado pelo pino (à esquerda); (b) meio da ZM. $\alpha$, ferrita alotriomorfa; $\alpha_{W}$, ferrita de Widmanstätten; $B$, bainita; $B_{C}$, bainita coalescida; $M$, martensita; $M A$, martensita-austenita. Velocidade de rotação $2000 \mathrm{rpm}$. MEV, ataque com Nital $1 \%$.

A quantidade e o tamanho da bainita coalescida diminuíram com o aumento da velocidade de rotação, como indicado na Figura 6, o que pode ser explicado a partir das seguintes considerações: primeiro, como um dos "requisitos" para a formação da bainita coalescida é um alto super-resfriamento [32, 33], é esperado que o super-resfriamento na menor velocidade de rotação de ferramenta aqui empregado seja maior do que nas outras condições (porque o aporte de energia, teoricamente, foi menor). Segundo, como a quantidade de ferrita alotriomorfa aumenta com o aumento da velocidade de rotação e/ou tempo de mistura, menor quantidade de bainita (e também de martensita) é formada. No presente estudo, acredita-se que a deformação no processo também contribua para a formação da bainita coalescida, seja pela formação de maior quantidade de placas com mesma orientação, ou por aproximar as temperaturas $B_{S}$ e $\mathrm{M}_{\mathrm{S}}$ (alguns estudos mostraram que a aplicação de deformação diminui a temperatura $\mathrm{B}_{\mathrm{S}}$ [34-37].

Na ZTMA, a microestrutura resultante era composta por ferrita, austenita, bainita e martensita, como mostrado na Figura 7, o que indica que a temperatura atingida estava entre $A c_{1}$ e $\mathrm{Ac}_{3}$, onde ferrita e austenita coexistem. Durante o resfriamento, houve a transformação de alguns grãos de austenita, os maiores, 
em bainita e martensita. Isto pode ser atribuído à estabilização química dos menores grãos de austenita, que possuem maior teor de carbono e, portanto, menor temperatura $\mathrm{M}_{\mathrm{S}}[38]$. Além disso, a deformação moderada nesta região pode ter contribuído para

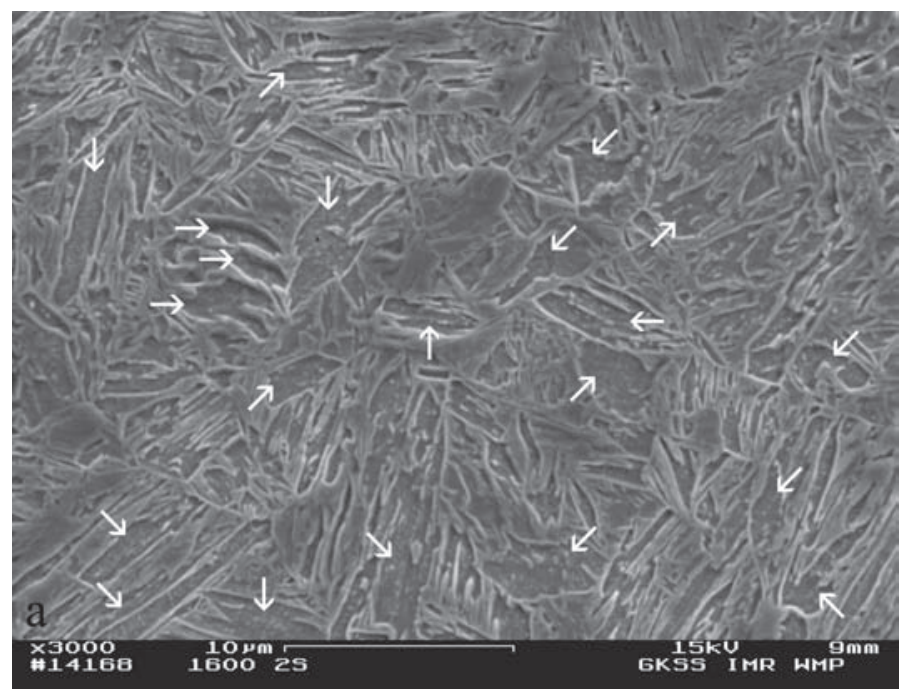

a estabilização mecânica da austenita: grãos menores possuem maiores densidades de discordâncias, com maiores energias de falha de empilhamento associadas, resultando em menor forçamotriz para a transformação martensítica [39].

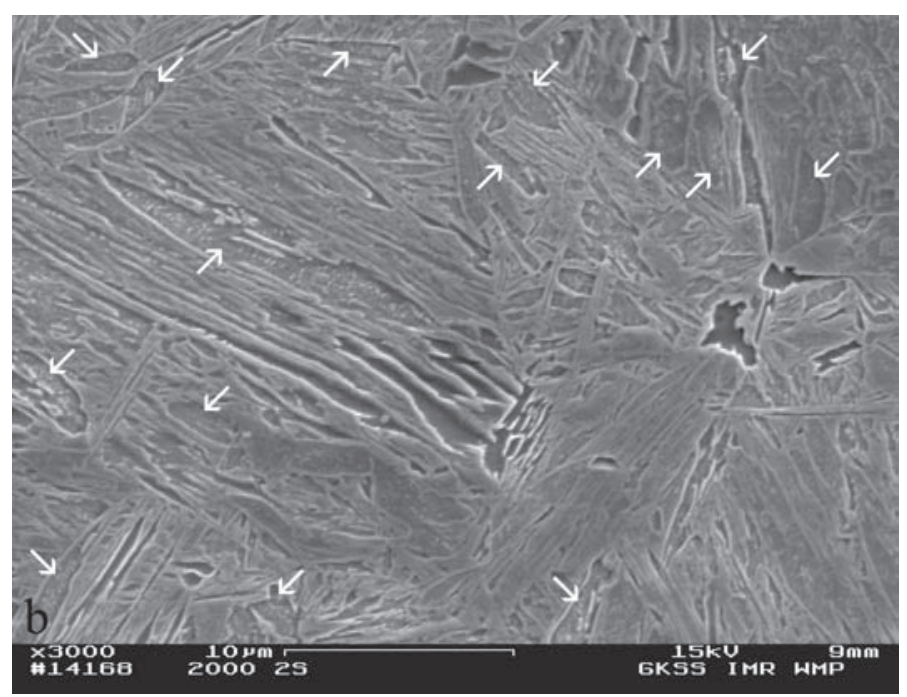

Figura 6. Comparação (visual) da quantidade e tamanho da bainita coalescida formada (algumas indicadas por flechas) no meio da ZM em amostras soldadas com velocidades de (a) $1600 \mathrm{rpm}$ e (b) $2000 \mathrm{rpm}$. MEV, ataque com Nital $1 \%$.

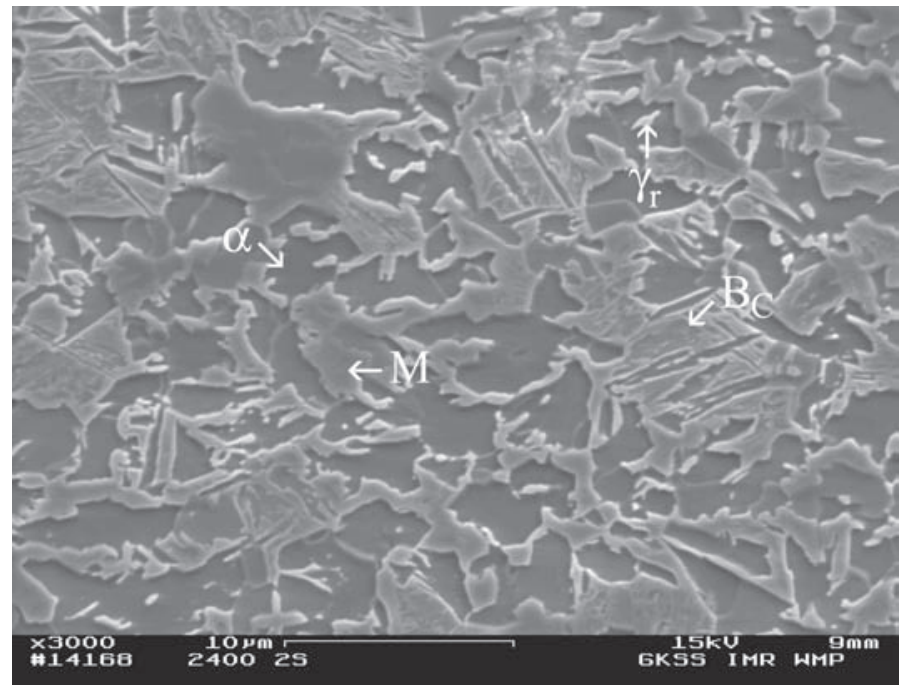

Figura 7. Microestrutura típica na ZTMA. $\alpha$, ferrita;

$B_{C}$, bainita coalescida; $M$, martensita; $\gamma_{r}$, austenita retida. Velocidade de rotação $2400 \mathrm{rpm}$. MEV, ataque com Nital $1 \%$.

A temperatura atingida na subzona ZTA1, assim como na ZTMA, provocou a formação de uma mistura de ferrita e austenita durante o aquecimento. Entretanto, nesta subzona a microestrutura não foi afetada pela deformação. Após resfriamento, a microestrutura era composta basicamente por ferrita e austenita, como pode ser visto na Figura 8(a). Na ZTA2 a temperatura atingida foi menor do que $\mathrm{Ac}_{1}$. Nesta subzona, como pode ser observado na Figura 8(b), houve transformação parcial da austenita em bainita, sendo que isto aconteceu de modo mais acentuado em maiores velocidades de rotação. Isto era esperado, pois nestas condições supõe-se que a taxa de resfriamento foi menor, ou seja, o material ficou exposto por maior tempo à faixa de temperaturas de formação da bainita. Estes resultados estão de acordo com os encontrados por Zaefferer et al. [40] e Jacques [41], onde maiores frações de bainita eram formadas em aços TRIP 800 com o aumento do tempo ou temperatura durante tratamento isotérmico.

Os perfis de microdureza resultantes mostraram que os maiores valores de dureza em cada zona/subzona foram encontrados na menor velocidade de rotação, como pode ser observado na Figura 9. Houve maior variação nos valores de dureza na ZM para a velocidade de rotação de $2400 \mathrm{rpm}$, devido a identações realizadas em áreas com maior quantidade de ferrita alotriomorfa, menos dura, ou de bainita ou martensita, mais duras.

A resistência das juntas, avaliada através de ensaios de cisalhamento, é mostrada na Figura 10, onde também está indicado o valor mínimo de pico de força recomendado para juntas produzidas através de soldagem a ponto por resistência elétrica, de acordo com a norma AWS D8.1M [42]. Considerando o valor médio de três corpos de prova para cada velocidade de rotação empregada, enquanto que o valor mínimo de acordo com a norma, de $5,82 \mathrm{kN}$, foi atingido para as velocidades de 2000 e $2400 \mathrm{rpm}$, o mesmo não aconteceu na velocidade de 1600 rpm, que apresentou o valor médio de 4,83 kN (mas deve ser salientado que, como mostrado na Figura 10, o desvio-padrão negativo na velocidade de $2400 \mathrm{rpm}$ ficou abaixo do especificado pela norma). Com o aumento da velocidade de rotação de 1600 rpm para $2000 \mathrm{rpm}$ houve um aumento de 40,9\% no valor médio da força máxima em cisalhamento. Porém, o aumento da velocidade de $2000 \mathrm{rpm}$ para $2400 \mathrm{rpm}$ ocasionou uma queda no valor médio de força em 9,4\%. Entretanto, independente da velocidade de rotação empregada, o mecanismo de fratura predominante em todas as amostras testadas foi dúctil, como mostrado na Figura 11. 

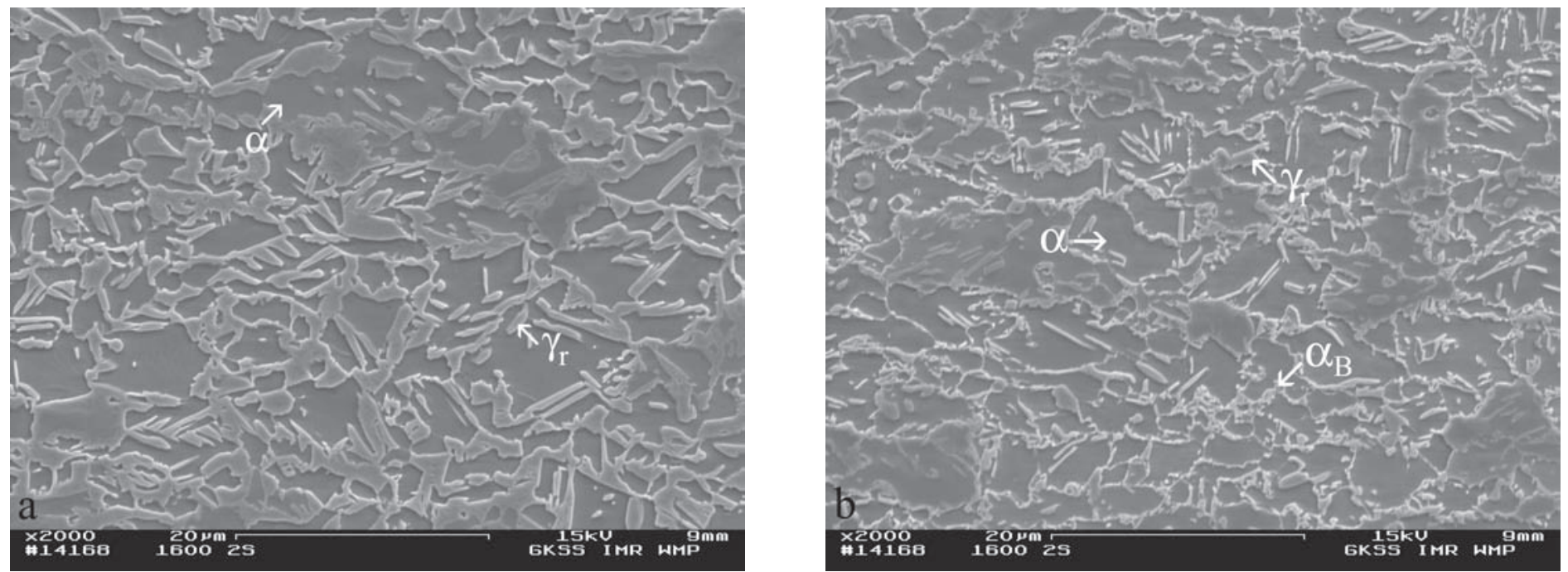

Figura 8. Microestruturas na ZTA: (a) ZTA1; (b) ZTA2. $\alpha$, ferrita; $\alpha_{B}$, bainita; $\gamma_{r}$, austenita retida. Velocidade de rotação $1600 \mathrm{rpm}$. MEV, ataque com Nital $1 \%$.

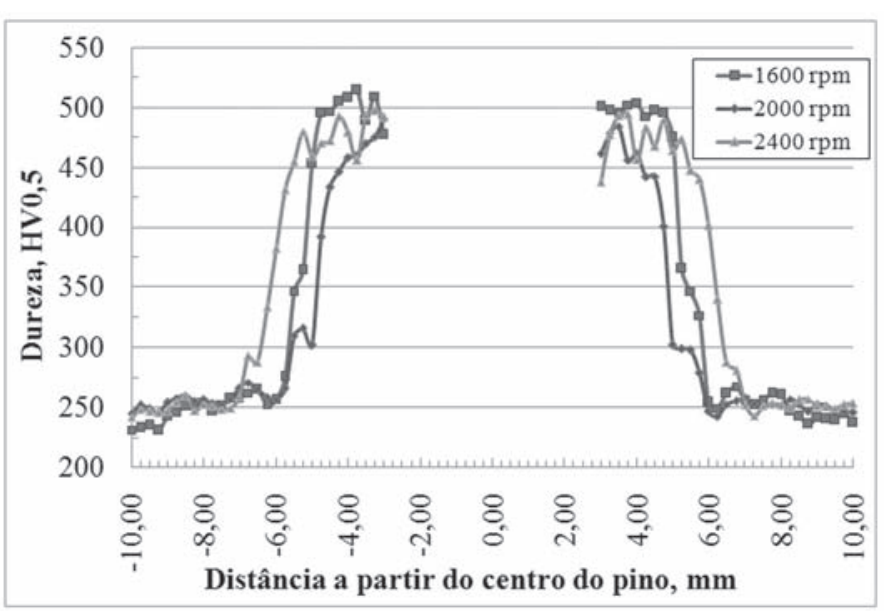

Figura 9. Perfis de microdurezas obtidos de acordo com a velocidade de rotação utilizada.

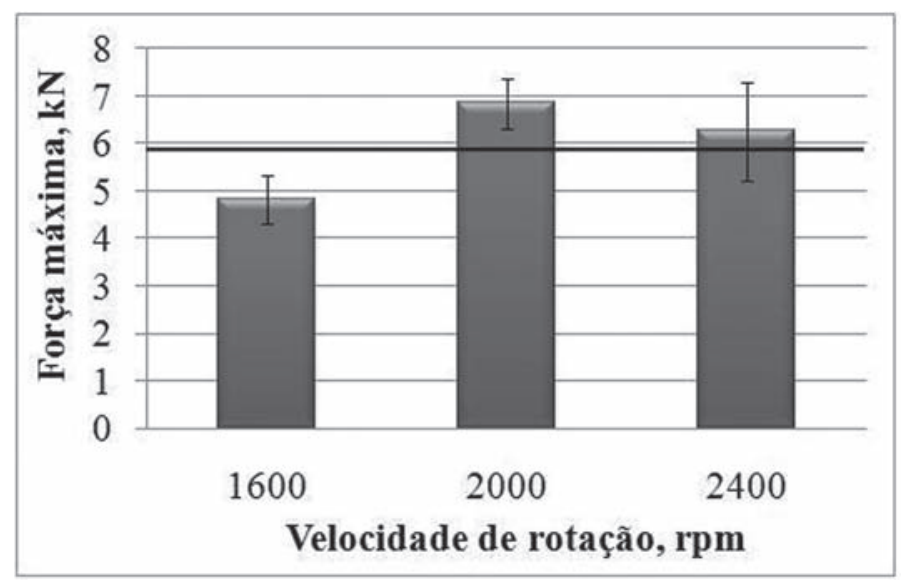

Figura 10. Valores de força máxima no ensaio de cisalhamento apresentados por cada velocidade de rotação, mostrando também o valor recomendado para soldas a ponto por resistência elétrica (linha horizontal). Média de três corpos de prova para cada condição.

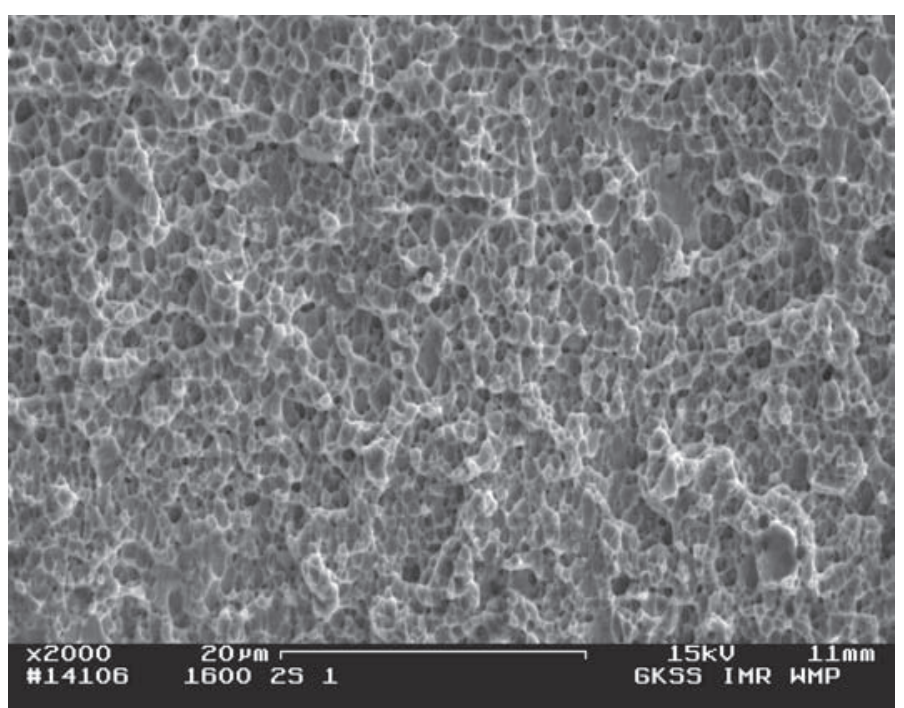

Figura 11. Mecanismo de fratura dúctil observado na amostra que apresentou menor valor de pico de força durante ensaios de cisalhamento, soldada a $1600 \mathrm{rpm}$. MEV.

As seções transversais das amostras testadas em cisalhamento podem ser observadas na Figura 12. Note-se que não existe um padrão de geometria de fratura para amostras com melhor ou pior desempenho em cisalhamento; apesar das amostras (e) e (f) apresentarem geometrias e valores semelhantes, as amostras (c) e (d) apresentaram valores semelhantes, mas possuem geometrias diferentes. O mesmo se aplica para as amostras, (c) e (e) (ou (f)), que possuem geometrias similares, mas seus valores são bem diferentes. 

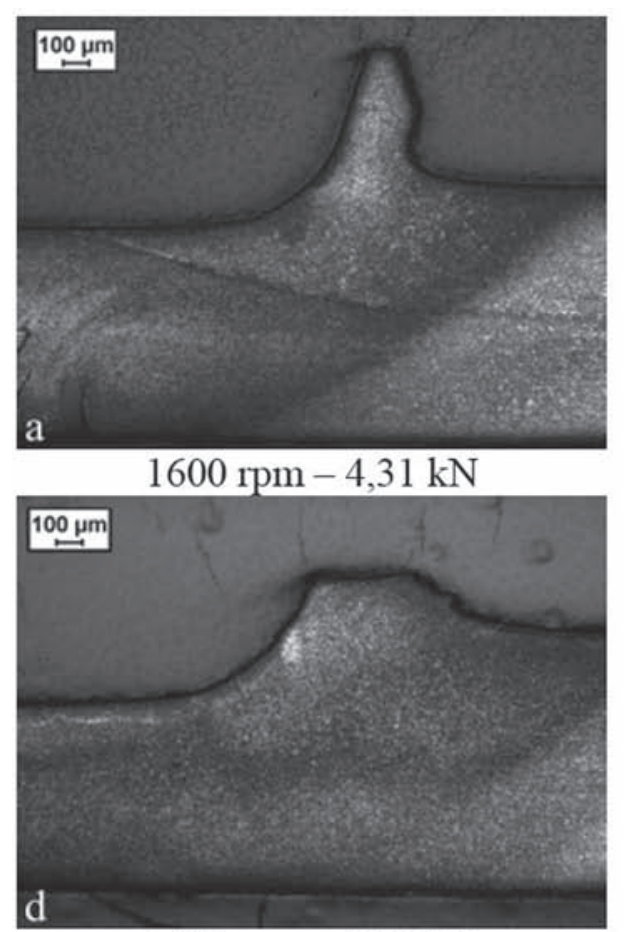

$1600 \mathrm{rpm}-5,33 \mathrm{kN}$
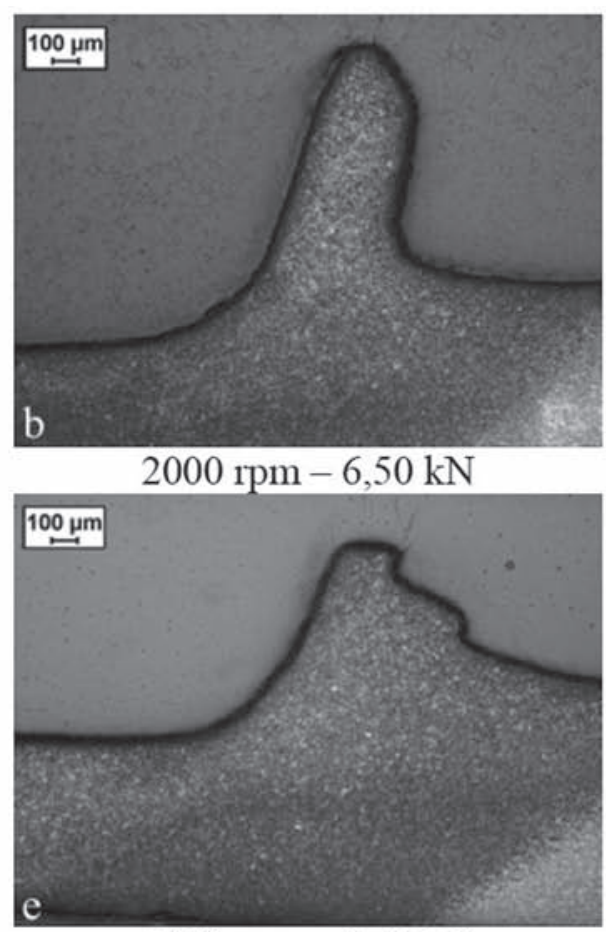

$2000 \mathrm{rpm}-7,46 \mathrm{kN}$

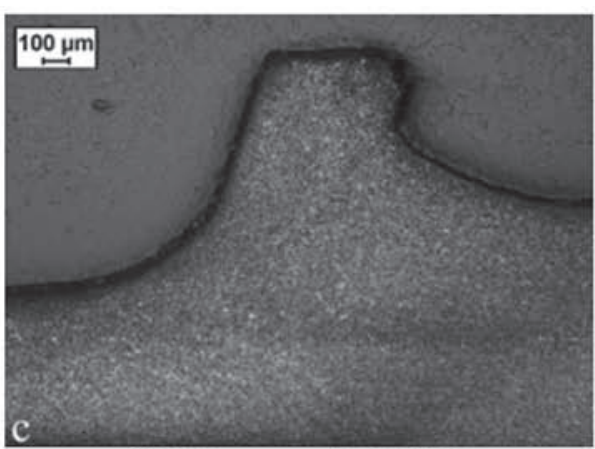

$2400 \mathrm{rpm}-5,26 \mathrm{kN}$

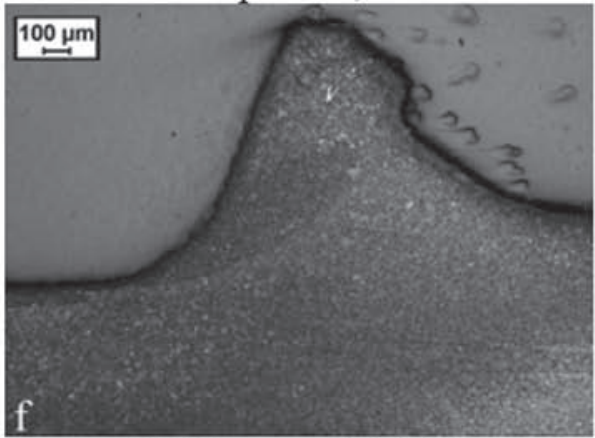

$2400 \mathrm{rpm}-7,44 \mathrm{kN}$

Figura 12. Seções transversais às fraturas de amostras testadas em cisalhamento. Para cada velocidade, são mostradas as amostras que apresentaram menor e maior valor de força no ensaio. MO, ataque com LePera.

A comparação entre os valores de força máxima obtidos no ensaio de cisalhamento e a DPZ, constante na Tabela 2, mostra que para os parâmetros de soldagem utilizados neste estudo existe uma correlação entre o CLL, a DPZ e a resistência da junta, ou seja, aumentando o CLL e a DPZ, houve um aumento no pico de força durante o ensaio de cisalhamento. No entanto, observa-se que a diferença entre valores de DPZ empregando as velocidades de rotação de 1600 e 2400 rpm é pequena (em torno de apenas

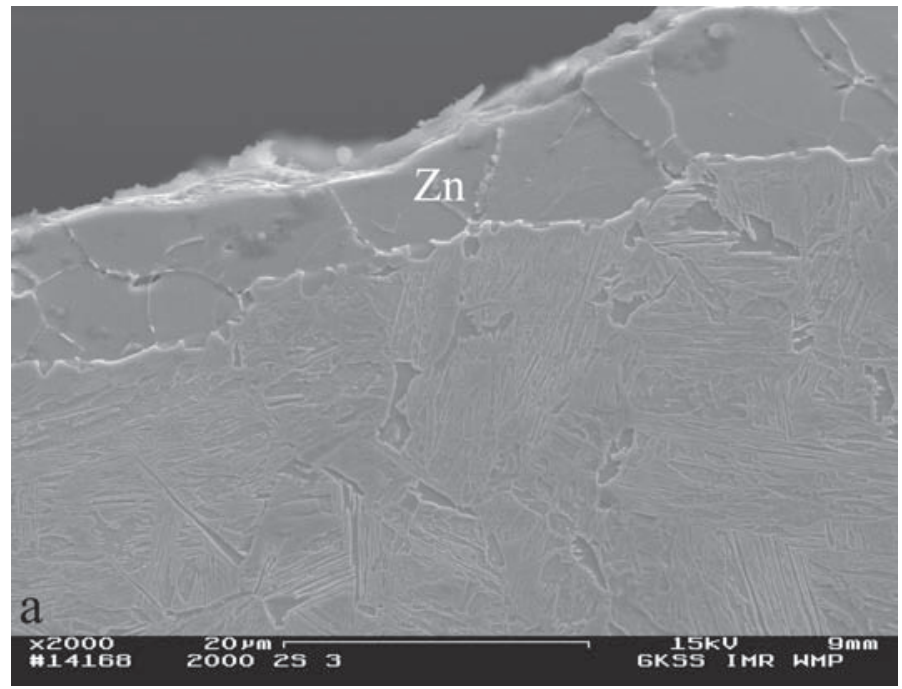

$20 \mu \mathrm{m})$, porém a média dos valores em cisalhamento são bem diferentes, 4,83 e 6,26 kN, respectivamente. Este fato, aliado à não-existência de um padrão de geometria de fratura reforça a idéia de que a resistência das juntas deve estar relacionada não só à DPZ, mas também ao CLL e certamente à microestrutura na ZM, próxima ao furo deixado pelo pino, em cada condição de soldagem.

A análise das seções transversais no MEV mostrou que as

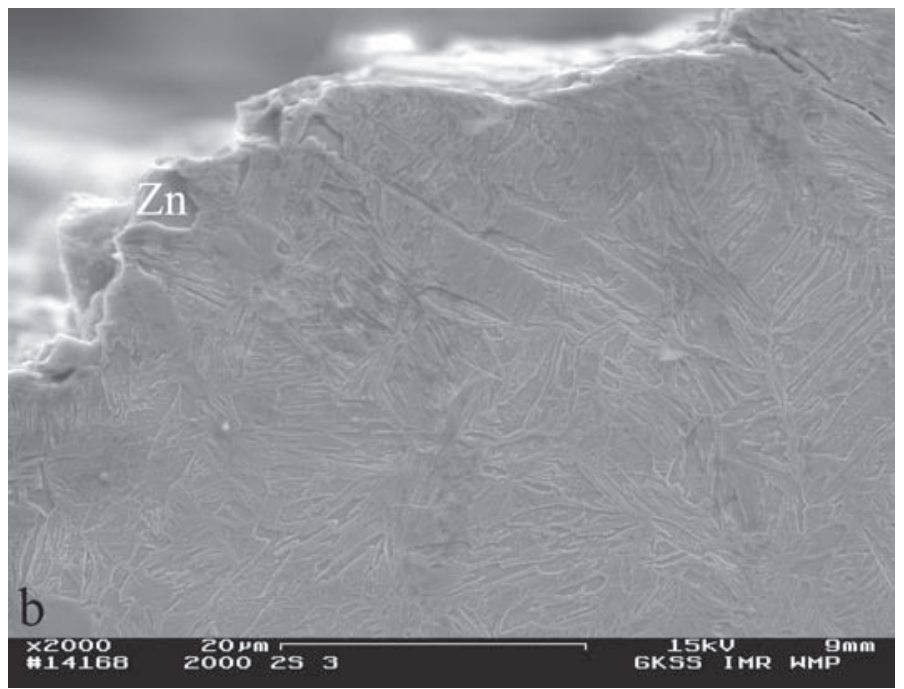

Figura 13. Imagens realizadas no MEV mostrando: (a) propagação da trinca ao longo da linha de zinco, sem nenhuma influência da microestrutura; (b) quando a linha de zinco termina, a trinca segue pela microestrutura. Velocidade de rotação 2000 rpm, ataque

Nital $1 \%$. 
fraturas em todas as condições de soldagem aqui empregadas ocorreram na ZM; elas iniciaram na interface entre as chapas superior e inferior, que atua como uma trinca, e propagaram ao longo da linha de zinco, como pode ser visto na Figura 13(a). Salienta-se que enquanto a trinca propagou por esta linha, a microestrutura ficou intacta, ou seja, não sofreu deformação. Porém, quando a linha de zinco deixou de existir, ou tornouse descontínua, a microestrutura influenciou no processo de fratura e ao final mostrou-se bastante deformada na direção do carregamento, como mostrado na Figura 13(b). Devido à deformação da microestrutura, não foi possível estabelecer a influência de cada fase nesta etapa final de fratura.

De acordo com os resultados acima, pressupõe-se que o processo de fratura nestas juntas é complexo e ocorreu seguindo as seguintes etapas:

1) a fratura iniciou na interface entre as chapas superior e inferior, na ZM, que atuou como uma trinca;

2) esta trinca propagou ao longo da linha de zinco, pois esta era a região de menor resistência na ZM;

3) após a trinca propagar por um certo período através da linha de zinco, existem duas possibilidades: ou o material rompeu porque a sua seção resistente tornou-se muito baixa ou, se a seção resistente ainda não tivesse atingido um valor crítico, houve influência da microestrutura presente nesta área na ruptura final da junta.

Com relação à última possibilidade, deve ser ressaltado que devido ao fluxo de material, pode ser que a linha de zinco na região um pouco mais próxima ao pino tornou-se descontínua, e então a microestrutura poder ter interagido com a trinca, facilitando ou retardando a sua propagação.

Sendo assim, pode-se dizer que os fatores desejáveis para a obtenção de juntas com boa resistência são:

- CLL grande, pois como a fratura começa na interface entre as chapas, um maior CLL significa que o caminho que a trinca terá que percorrer antes que a área resistente da junta atinja o valor crítico será maior;

- assim como o CLL, quanto maior a DPZ, melhor, com base nos mesmos motivos explicados acima;

- microestrutura na ZM, especialmente nesta região adjacente ao pino, com alta resistência.

Salienta-se que não existe necessariamente a influência preponderante de um destes fatores sobre a resistência das juntas, mas sim uma melhor combinação entre eles. No presente estudo, por exemplo, a melhor combinação entre CLL, DPZ e microestrutura resultante na ZM foi obtida na velocidade de $2000 \mathrm{rpm}$.

\section{Conclusões}

Juntas de um aço TRIP 800 foram produzidas através do processo de SPFMM, utilizando uma ferramenta feita em PCBN. Devido aos gradientes de temperatura e deformação impostos pelo processo, diferentes zonas foram formadas. $\mathrm{Na}$ ZM, a quantidade de ferrita alotriomorfa formada aumentou com o aumento da velocidade de rotação, enquanto que as quantidades de bainita coalescida e de martensita diminuíram, o que foi atribuído ao provável maior aporte de energia quando maiores velocidades de rotação foram utilizadas, levando a taxas de resfriamento mais lentas. Devido ao fluxo de material, formou-se uma linha de zinco na ZM. Quando as amostras foram submetidas ao carregamento em ensaios de cisalhamento, a fratura ocorreu seguindo esta linha de zinco, de forma que houve influência da microestrutura no processo de fratura apenas na sua etapa final, onde a linha de zinco não estava mais presente.

\section{Agradecimentos}

Os autores agradecem o suporte financeiro concedido pela CAPES e DAAD através do Projeto PROBRAL estabelecido entre Brasil e Alemanha, e ao GKSS Forschungszentrum GmbH pela infra-estrutura disponibilizada. Além disso, os autores agradecem ao CNPq pelas bolsas de doutorado concedidas.

\section{Referências Bibliográficas}

[1] BARON, J.; SHAW, J. Tool-and-die shops collaborate to help automotive OEMs and their suppliers form advanced highstrength steels. Disponível em <http://www.autosteel.org>. Acesso em: 05 out. 2007.

[2] FURUSAKO, S.; MIYAZAKI; Y. Improvement of crashworthiness by application of high-strength steel, Nippon Steel Technical Report, n. 95, p. 35-38, 2007.

[3] DE COOMAN, B.C. Structure-properties relationship in TRIP steels containing carbide-free bainite, Current Opinion in Solid State \& Materials Science, v. 8, p.285-303, 2004.

[4] JACQUES, P.J. et al. The developments of cold-rolled TRIPassisted multiphase steels. Al-alloyed TRIP-assisted steels. ISIJ International, v. 41, n. 9, p. 1068-1074, 2001.

[5] SRIVASTAVA, A.K., et al. Effect of heat treatment on microstructure and mechanical properties of cold rolled C-MnSi TRIP-aided steel. Materials Characterization, v. 57, p. 127135, 2006.

[6] KIM, S.J., et al. Effects of heat treatment and alloying elements on the microstructures and mechanical properties of 0.15 wt pct $\mathrm{C}$ transformation-induced plasticity-aided cold rolled steel sheets. Metallurgical and Materials Transactions A, v. 32, n. 3, p. 505-514, 2001.

[7] SRIVASTAVA, A.K. et al. Microstructural and mechanical characterization of C-Mn-Al-Si cold-rolled TRIP-aided steel, Materials Science and Engineering A, v. 445-446, p. 549-557, 2007.

[8] KANEKO, Y. et al. Material characteristics of TRIP steel with self-diagnosis and application to structural systems. Smart Materials Structures, v. 16, p. 2464-2476, 2007.

[9] SHI, G.; WESTGATE, S. Techniques for improving the weldability of TRIP steel using resistance spot welding. Disponível em <http://www.msm.cam.ac.uk/phase-trans/2005/ LINK/89.pdf>. Acesso em 24 set. 2007.

[10] HAFERKAMP, H.; MEIER, O.; HARLEY, K. Laser beam welding of new high strength steels for auto body construction, 
Key Engineering Materials, v. 344, p. 723-730, 2007.

[11] WESLING et al., Widerstandspunktschweißen moderner Mehrphasenstähle, TUC Forschung-Stähle, n. 16, p.54-58, 2005.

[12] WILLIAMS, N.T.; PARKER, J.D. Review of resistance spot welding of steel sheets - part 2: factors influencing electrode life, International Materials Reviews, v. 49, n. 2, p. 77-108, 2004.

[13] MÜFTÜO LU, F.; KESKINEL, T. Effect of coating thickness on electrode life in spot welding of galvanized steels, Turkish Journal of Engineering and Environmental Sciences, v. 31, p. 183-187, 2007.

[14] DANESHPOUR, S., et al. Failure behaviour of laser spot welds of TRIP800 steel sheets under coach-peel loading. Science and Technology of Welding and Joining, v. 12, n. 6, p. 508-515, 2007.

[15] MAZZAFERRO, C.C.P. et al. Friction stir spot welding of a TRIP 800 steel. In: INTERNATIONAL SEMINAR ON FRICTION STIR WELDING OF STEELS, 2007, Geesthacht, Proceedings... Alemanha, 2007.

[16] Mazda develops world's first aluminum joining technology using friction heat. Disponível em <http://www.mazda.com/ publicity/release/2003/200302/0227e.html>. Acesso em 09 nov. 2007.

[17] KHAN, M.I., et al. Resistance and friction stir spot welding of DP600: a comparative study, Science and Technology of Welding and Joining, v. 12, n. 2, p. 175-182, 2007.

[18] KYFFIN, W.J., et al. Recent developments in friction stir spot welding of automotive steels. In: SHEET METAL WELDING CONFERENCE, XII, 2006, Livinoia, Proceedings... USA: Michigan, 2006.

[19] KYFFIN, W.J., et al. Progress in FSSW of DP 800 high strength automotive steel. In: INTERNATIONAL SYMPOSIUM OF FRICTION STIR WELDING, 6º 2006, Proceedings... Canada: Quebec, 2006.

[20] FENG, Z., et al. Friction stir spot welding of advanced high-strength steels: a feasibility study, SAE Technical Paper 2005-01-1248. 2005.

[21] MAZZAFERRO, C.C.P. Soldagem a ponto por fricção e mistura mecânica de um aço TRIP 800: processo, microestrutura e propriedades. Tese de Doutorado. Universidade Federal do Rio Grande do Sul, 2008.

[22] GIRAULT, E., JACQUES, P., HARLET, P., MOLS, K., VAN HUMBEECK, J., AERNOUDT, E., DELANNAY, F. Metallographic methods for revealing the multiphase microstructure ofTRIP-assisted steels, MaterialsCharacterization, v. 40, n. 2, p. 111-118, 1998.

[23] MODENESI, P.J. Soldabilidade dos aços transformáveis. Disponível em <http://www.demet.ufmg.br/labs/soldagem/ textos/metferritic.pdf>. Acesso em 30 maio 2008.

[24] INTERNATIONAL ORGANIZATION FOR STANDARDIZATION ISO 14273 (E): Specimen dimensions and procedure for shear testing resistance spot, seam and embossed projection welds. 2000, 8p.

[25] KYFFIN, W.J., et al. Progress in FSSW of DP 800 high strength automotive steel. Proceeding of the 6th International Symposium of Friction Stir Welding. 2006, Canada.

[26] SANTELLA, M.L., et al. Friction-stir spot-welding of highstrength steel. FY 2006 Progress Report. Disponível em <http:// www 1.eere.energy.gov/vehiclesandfuels/pdfs/alm_06/5d_ santella.pdf>. Acesso em: 12 jun. 2008.

[27] BHADESHIA, H. K. D. H.: in 'Mathematical modelling of weld phenomena' (ed. H. Cerjak and H. K. D. H. Bhadeshia), 71-118; 1995, Institute of Materials, London.

[28] HANLON, D.N.; SIETSMA, J.; VAN DER ZWAAG, S. The effect of plastic deformation of austenite on the kinetics of subsequent ferrite formation, ISIJ International, v. 41, p. 10281036, 2001.

[29] RYU, H.-B.; SPEER, J.G.; WISE, J.P. Effect of thermomechanical processing on the retained austenite content in a Si-Mn transformation induced plasticity steel, Metallurgical and Materials Transactions A, p. 2811-2816, 2002.

[30] HONG, S.C., et al. Effect of undercooling of austenite on strain induced ferrite transformation behavior, ISIJ International, v. 43, n. 3, p. 394-399, 2003.

[31] LIU, X.; SOLBERG, J.K.; JENGEDAL, R. Measurement of austenite-to-ferrite transformation temperature after multipass deformation of steels, Materials Science and Engineering A, v. 194, p. 15-18, 1995.

[32] BHADESHIA, K.H.D.H., et al. Coalesced bainite, Transactions of the Indian Institute of Metals, v. 59, p. 689-694, 2006.

[33] KEEHAN, E., et al. New developments with C-Mn-Ni high-strength steel weld metals, Part A - microstructure. Welding Journal, v. 85, p. 200s-210s, 2006.

[34] BHADESHIA, H.K.D.H. Bainite in Steels. London: Institute of Materials, 2001.

[35] LARN, R.H.; YANG, J.R. The effect of compressive deformation of austenite on the bainitic ferrite transformations in Fe-Mn-Si-C steels, Materials Science and Engineering A, v. 278, p. 278-291, 2000.

[36] BHADESHIA, H.K.D.H. Possible effects of stress on steel weld microstructures. Mathematical Modelling of Weld Phenomena. Institute of Materials, London, 1995, p. 71-118.

[37] JANDOVÁ, D., et al. Microstructure and phase transformations in alloy steels and simulations of their processing, AEDS Workshop, 2004, Pilsen, Czech Republic. Disponível em <http://www.kks.zcu.cz/aeds/workshop/2004/fullpapers/113. pdf>. Acesso em: 09 de nov. 2007.

[38] JUN, J.-H.; CHOI, C.-S. Variation of stacking fault energy with austenite grain size and its effect on the $\mathrm{M}_{\mathrm{s}}$ temperature of $\gamma \rightarrow \varepsilon$ martensitic transformation in Fe-Mn alloys, Materials Science and Engineering A, v. A257, p. 353-356, 1998.

[39] WAN, J.; CHEN, S.; HSU, T.Y. Journal of Physics IV, v. 112, p. 381-384, 2003.

[40] ZAEFFERER, S.; OHLERT, J.; BLECK, W. A study of microstructure, transformation mechanisms and correlation between microstructure and properties of a low alloyed TRIP steel. Acta Materialia, v. 52, p. 2765-2778, 2004. 
[41] JACQUES, P.J., et al. Multiscale mechanics of TRIPassisted multiphase steels: I. characterization and mechanical testing. Acta Materialia, v. 55, p. 3681-3693, 2007.

[42] AMERICAN WELDING SOCIETY AWS D8.1M: Specification for automotive weld quality - resistance spot welding of steel. 2007, 28p. 